Wolf-Michael Kähler

SPSS für Windows 


\section{Lehrbücher Informatik}

Regelbasierte Inferpolation und Fuzzy Control von Dirk Drechsel

\section{Neuronale Nekze und Fuzzy-Systeme}

Grundlagen des Konnektionismus, Neuronaler Fuzzy-Systeme und der Kopplung mit wissensbasierten Methoden

von D. Nauck, F. Klawonn, R. Kruse

Einführung in die Statistische Datenanalyse

Grundlegende Verfahren und deren EDV-gestützter Einsatz von Wolf-Michael Kähler

\section{SPSS für Windows}

Eine Einführung in die Datenanalyse für

die aktuellen SPSS-Versionen

von Wolf-Michael Kähler

\section{SPSS for UNIX}

Datenanalyse unter UNIX von Wolf-Michael Kähler

Professionelles Datenbank-Design mit ACCESS 2.0

von Ernst Tiemeyer u. Klemens Konopasek

DERIVE für den Mathematik-Unterricht

Unter DOS und Windows

von Wolfram Koepf

Algorithmische lineare Algebra

Eine Einführung für Mathematiker und Informatiker von Herbert Möller

\section{Programmieren mit Fortran 90}

Eine umfassende Einführung

von Hans-Pełer Bäumer

\section{Excel für Techniker und lngenieure}

Eine grundlegende Einführung am Beispiel technischer Problemstellungen von Hans-Jürgen Holland und Frank Bracke

\section{Vieweg}


Wolf-Michael Kähler

\section{SPSS für Windows}

Eine Einführung in die Datenanalyse für die aktuellen Versionen

3., erweiterte und überarbeitete Auflage 
1. Auflage 1994

2., verbesserte und erweiterte Auflage 1994

3., erweiterte und überarbeitete Auflage 1996

Alle Rechte vorbehalten

() Springer Fachmedien Wiesbaden 1996

Ursprünglich erschienen bei Friedr. Vieweg \& Sohn Verlagsgesellschaft mbH, Braunschweig/Wiesbaden, 1996

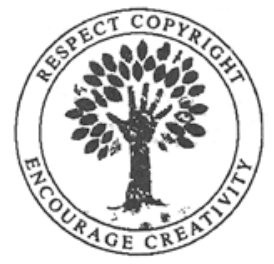

Das Werk einschließlich aller seiner Teile ist urheberrechtlich geschützt. Jede Verwertung außerhalb der engen Grenzen des Urheberrechtsgesetzes ist ohne Zustimmung des Verlags unzulässig und strafbar. Das gilt insbesondere für Vervielfältigungen, Übersetzungen, Mikroverfilmungen und die Einspeicherung und Verarbeitung in elektronischen Systemen. 
für meine Tochter Iris

\section{Vorwort zur 3. Auflage}

Dieses Buch wendet sich an Leser, die empirisch erhobenes Datenmaterial mit einem PC unter Windows statistisch auswerten und dabei das Programmsystem "SPSS für Windows" zur statistischen Datenanalyse einsetzen wollen. Die Darstellung ist so gehalten, daß keine besonderen Vorkenntnisse aus dem Bereich der Elektronischen Datenverarbeitung vorausgesetzt werden. Vielmehr soll der Leser in einfacher Weise an das Werkzeug "SPSS für Windows" herangeführt und schnell in die Lage versetzt werden, Anforderungen zur statistischen Datenanalyse zu stellen.

Mit diesem Buch wird eine problembezogene Einführung und keine handbuchartige Aneinanderreihung der Leistungen des Programmsystems "SPSS für Windows" vorgelegt. Neben der Darstellung der möglichen Anforderungen wird - am Beispiel einer (einzigen) empirischen Untersuchung - die Form der von "SPSS für Windows" ausgegebenen Analyseergebnisse erläutert und die daraus resultierende Interpretation angegeben. Dabei wird unterstellt, daß der Leser über Grundkenntnisse in Statistik verfügt.

Aufgrund der in Projektberatungen und in Lehrveranstaltungen gesammelten Erfahrungen ist dieses Buch, das den Leistungsumfang des Basispakets "Basics" beschreibt, so konzipiert, daß es sowohl als Begleitlektüre für Lehrveranstaltungen als auch zum Selbststudium geeignet ist.

Gegenüber der "Programmversion 6", deren Leistungsumfang Gegenstand der Beschreibung im Rahmen der 2. Auflage war, haben sich mit der zwischenzeitlich angebotenen "Programmversion 7" weitere Möglichkeiten für den Anwender von "SPSS für Windows" eröffnet.

In dieser Hinsicht ist nicht nur die Neugestaltung der Ausgabe von angeforderten Analyseergebnissen und deren Verwaltung zu nennen, sondern es ist 
auch der grundlegenden Neukonzeption der Dialogführung - im Hinblick auf die neuerdings verwendeten "Windows" - Rechnung zu tragen.

Im Zuge der innovativen Weiterentwicklung von "SPSS für Windows" kommt den heutzutage aktuellen Windows-Umgebungen eine besondere Bedeutung zu, da nur sie die Plattformen für einen leistungsfähigen Einsatz dieses Datenanalysesystems in der aktuellen Version 7 bieten. Im Hinblick auf diesen Sachverhalt stellt dieses Buch eine plattformübergreifende Beschreibung für das Arbeiten mit "SPSS für Windows" dar.

Der Firma SPSS GmbH Software danke ich für ihre freundliche Unterstützung und dem Vieweg Verlag für die traditionell gute Zusammenarbeit.

Ritterhude, im Oktober 1996

Wolf-Michael Kähler 


\section{Inhaltsverzeichnis}

1 Vorbereitungen zur Datenanalyse 1

1.1 Beispiel einer empirischen Untersuchung . . . . . . . . . 1

1.2 Einsatz von "SPSS für Windows" . . . . . . . . . . . 2

1.3 Kodierung und Strukturierung der Daten . . . . . . . . . 4

1.3.1 Kodeplan . . . . . . . . . . . . . . . 4

1.3.2 Kodierung von fehlenden Werten . . . . . . . . . 5

1.3.3 Zusammenstellung von Daten in einer Daten-Tabelle . 6

1.3.4 Die SPSS-Daten-Tabelle . . . . . . . . . . . . . 7

1.4 Datenerfassung . . . . . . . . . . . . . 8

1.4.1 Beginn des Dialogs mit dem SPSS-System . . . . . . 8

1.4.2 Dialog im "Daten-Editor-Window" . . . . . . . . . 12

1.4.3 Datensicherung in eine SPSS-Datendatei . . . . . . 19

1.4 .4 Dialogende . . . . . . . . . . . . . . 24

1.4.5 Fortsetzung der Datenerfassung . . . . . . . . . . 25

1.5 Meßniveau der Merkmale . . . . . . . . . . . 27

2 Datenanalyse mit dem SPSS-System 29

2.1 Eine Häufigkeitsauszählung . . . . . . . . . . . . . 29

2.2 Das "Output-Navigator-Window" . . . . . . . . . . . 38

2.2.1 Die Gliederung des "Output-Navigator-Windows" . . 39

2.2.2 Positionierung innerhalb des "Output-Navigator-Windows" . . . . . . . . . . . . . . 40

2.2.3 Änderung des Inhalts des "Output-Navigator-Windows" 41

2.2.4 Ausgabe des Inhalts des "Output-Navigator-Windows" 45

2.3 Fortsetzung des Dialogs . . . . . . . . . . . 47 
2.4 SPSS-Kommandos . . . . . . . . . . . . . . . . . 48

2.4.1 Beispiele für SPSS-Kommandos . . . . . . . . . . 48

2.4.2 Aufbau und Ausführung von SPSS-Kommandos . . 49

2.4.3 Syntax von SPSS-Kommandos . . . . . . . . . 53

2.4.4 Anzeige der Syntax eines SPSS-Kommandos . . . . . . 54

2.4.5 Kommentierung von SPSS-Kommandos . . . . . . 56

2.5 Die Journal-Datei . . . . . . . . . . . . . . . . 56

3 Veränderung und Ergänzung der SPSS-Daten-Tabelle 59

3.1 Änderung von Variablennamen . . . . . . . . . . . . . . 59

3.2 Numerische und alphanumerische Variablen . . . . . . . . 61

3.3 Variablen- und Werteetiketten . . . . . . . . . . . . 64

3.4 Missing values . . . . . . . . . . . . . . 68

3.4.1 Benutzerseitig festgelegte missing values . . . . . . . 68

3.4.2 Der system-missing value . . . . . . . . . . . 69

3.5 Modifikation der SPSS-Daten-Tabelle . . . . . . . . . . 70

3.5.1 Rekodierung . . . . . . . . . . . . 70

3.5.2 Automatische Rekodierung . . . . . . . . . . 75

3.6 Überprüfung der Eingabedaten . . . . . . . . . . . . 77

3.6.1 Eingabefehler . . . . . . . . . . . . . 77

3.6.2 Überprüfung von Werten . . . . . . . . . . 79

3.6.3 Anzeige von Werten . . . . . . . . . . . . 81

3.7 Inhalt der SPSS-Daten-Tabelle . . . . . . . . . . . 85

3.8 Vereinbarung und Aktivierung von Variablen-Sets . . . . . . 88

4 Beschreibung von Merkmalen $\quad 91$

4.1 Häufigkeitsverteilungen und Statistiken . . . . . . . . . 91

4.1.1 Ausgabe von Häufigkeitsverteilungen . . . . . . . . . 91

4.1.2 Steuerung der Ausgabe . . . . . . . . . . . 93

4.1.3 Berechnung von Statistiken . . . . . . . . . . 95

4.1.3.1 Die Berechnung von Perzentilwerten . . . . . . . 9 95

4.1.3.2 Maße der zentralen Tendenz . . . . . . . . . . . . 97

4.1.3.3 Maße der Variabilität . . . . . . . . . . . . 98

4.1.3.4 Maße der Wölbung und der Schiefe . . . . . . . . . 99 
4.1.3.5 Maß für die Schätzgüte . . . . . . . . . . 100

4.1.3.6 Gruppierte Daten . . . . . . . . . . . . . . 101

4.1.4 Anzeige von Balkendiagrammen und Histogrammen . 102

4.1.5 Ausgabe von Grafiken . . . . . . . . . . . . . . 103

4.1.6 Berechnung von Statistiken

für kontinuierliche Merkmale . . . . . . . . . . . . 104

4.2 Die Subfile-Struktur . . . . . . . . . . . . . . 108

4.3 Sortierung der SPSS-Daten-Tabelle . . . . . . . . . . . 110

4.4 Beschreibung von Merkmalen durch einen Report . . . . . . . 113

4.4.1 Break- und Kolumnen-Variablen . . . . . . . . . 113

4.4.2 Ausgabe von Statistiken . . . . . . . . . . . . 119

4.4.3 Textausgabe in Kopf- und Fußzeilenbereiche . . . . . . 121

4.4.4 Gestaltung der Reportausgabe . . . . . . . . . 123

4.4.5 Verrechnung von missing values . . . . . . . . . 126

4.4.6 Report-Struktur bei mehreren Break-Variablen . . . 127

4.4.7 Kolumnen-orientierte Reports . . . . . . . . . . . . . 128

4.4.8 Aggregation über Kolumnen . . . . . . . . . . . . 134

4.5 Vereinfachte Reportausgabe für intervallskalierte Merkmale . 137

4.6 Häufigkeitsauszählung bei Mehrfachnennungen . . . . . . . 142

4.7 Explorative Datenanalyse . . . . . . . . . . . . . . 148

4.7.1 Statistiken und Extremwerte . . . . . . . . . 148

4.7.2 Schätzung der zentralen Tendenz . . . . . . . . . 150

4.7.3 Berechnung von Perzentilwerten . . . . . . . . 152

4.7.4 Beschreibung von Verteilungen durch Histogramme . . 152

4.7.5 "Stem-and-leaf"-Plots . . . . . . . . . . . . 153

4.7 .6 Boxplots . . . . . . . . . . . . . . . . 154

4.7.7 Überprüfung auf Normalverteilung . . . . . . . . . 156

4.7.8 Gruppenvergleiche . . . . . . . . . . . . 158

4.7.9 "Spread-and-level"-Plots . . . . . . . . . . . . 160

4.7.10 Behandlung von missing values . . . . . . . . . . 162 
5 Beschreibung der Beziehung von Merkmalen

5.1 Analyse von Kontingenz-Tabellen . . . . . . . . . . . 163

5.1.1 Die gemeinsame Häufigkeitsverteilung zweier Merkmale 163

5.1.2 Anforderung von Kontingenz-Tabellen . . . . . . . 165

5.1.3 Steuerung der Tabellenausgabe . . . . . . . . . . . 168

5.1.4 Statistischer Zusammenhang zwischen nominalskalierten Merkmalen . . . . . . . . . . . . 171

5.1.4.1 Anforderung von Statistiken . . . . . . . . . 171

5.1 .4 .2 Chi-Quadrat . . . . . . . . . . . . 175

5.1.4.3 Phi-Koeffizient . . . . . . . . . . . . . 175

5.1 .4 .4 Cramer's V . . . . . . . . . . . . . . 176

5.1.4.5 Kontingenzkoeffizient C . . . . . . . . . . 176

5.1.4.6 Der Likelihood-Quotienten-Chi-Quadrat-Wert . . . . 177

5.1.4.7 Das PRE-Maß Lambda . . . . . . . . . . . . . . . 177

5.1.4.8 Der Tau-Koeffizient von Goodman und Kruskal . . . 180

5.1.4.9 Cohen's Kappa . . . . . . . . . . . . . . . . . . . . 181

5.1.4.10Das relative Risiko . . . . . . . . . . . . . 181

5.1.5 Statistischer Zusammenhang zwischen ordinalskalierten Merkmalen . . . . . . . . . . . . 183

5.1.5.1 Anforderung von Statistiken . . . . . . . . . 183

5.1.5.2 Konkordante und diskordante Paare . . . . . . . 185

5.1.5.3 Positive und negative Beziehungen . . . . . . . 185

5.1.5.4 Der Gamma-Koeffizient . . . . . . . . . . . . 186

5.1.5.5 Der Koeffizient Somers' d . . . . . . . . . . . 187

5.1.5.6 Kendall's Tau $_{B}$ und $T a u_{C} \ldots \ldots \ldots$. . . . . 188

5.1.6 Statistischer Zusammenhang zwischen einem nominal- und einem intervallskalierten Merkmal . . . . 189

5.1.7 Inferenzstatistisches Schließen . . . . . . . . . . . 191

5.2 Beschreibung der Beziehung von intervallskalierten Merkmalen 195

5.2.1 Grafische Beschreibung . . . . . . . . . . 195

5.2.1.1 Ausgabe von Streudiagrammen . . . . . . . . 195

5.2.1.2 Identifikation von Cases . . . . . . . . . . . . . 197

5.2.1.3 Darstellung von unterschiedlichen Häufigkeiten . . . 200

5.2.1.4 Gestaltung des Layouts . . . . . . . . . . . 202 
5.2.1.5 Überlagerung von Streudiagrammen . . . . . . . . . 203

5.2.1.6 Optionale Anforderungen . . . . . . . . . . . . 204

5.2.1.7 Weitere Darstellungsformen von Streudiagrammen . . 205

5.2.2 Der Korrelationskoeffizient " $\mathrm{r}$ " von Bravais-Pearson . 208

5.3 Beschreibung der Beziehung von ordinalskalierten Merkmalen 214

5.3.1 Spearman's Rho . . . . . . . . . . . . . . . 214

5.3 .2 Kendall's $\operatorname{Tau}_{B} \ldots \ldots \ldots \ldots \ldots \ldots$

5.4 Drittvariablen-Kontrolle von statistischen Beziehungen . . . . 217

5.5 Vergleich von Mittelwerten . . . . . . . . . . . . 224

5.5.1 Prüfung eines Mittelwerts (t-Test) . . . . . . . 224

5.5.2 Der Koeffizient Eta-Quadrat . . . . . . . . . 226

5.5.3 Die Varianzanalyse-Tafel . . . . . . . . . . . . 227

5.5.4 Linearitäts-Test . . . . . . . . . . . . . . . . . 229

5.5.5 Mittelwertvergleich für zwei Gruppen . . . . . . . 231

5.5.5.1 Der t-Test für unabhängige Stichproben . . . . . . . 231

5.5.5.2 Der t-Test für abhängige Stichproben . . . . . . . 234

5.5.5.3 Optionale Anforderungen . . . . . . . . . 237

6 Veränderung der SPSS-Daten-Tabelle 239

6.1 Unbedingte Zuweisung . . . . . . . . . . . . . . 239

6.2 Bedingte Zuweisung . . . . . . . . . . . . . . . 248

6.3 Zuordnung von alphanumerischen Werten . . . . . . . 252

6.4 Auszählung von Werten . . . . . . . . . . . 256

6.5 Gewichtung von Cases . . . . . . . . . . . . . 259

6.6 Datenauswahl . . . . . . . . . . . . . . 262

6.6.1 Gezielte Auswahl von Cases . . . . . . . . . . . . 262

6.6.2 Zufällige Auswahl von Cases . . . . . . . . 266

7 Ausgabe des SPSS-Systems $\quad 269$

7.1 Text-Informationen . . . . . . . . . . . . . . . . 270

7.2 "Pivot-Tabellen"-Informationen . . . . . . . . . . . . 275

7.3 Druckausgabe . . . . . . . . . . . . . . . . 288 
8 Aufbau von Grafiken $\quad 295$

8.1 Anzeige auf dem Bildschirm . . . . . . . . . . . 295

8.1.1 Das Menü "Graphs" . . . . . . . . . . . . . . 295

8.1.2 Aufbau von Balkendiagrammen . . . . . . . . . . 296

8.1.3 Das "Chart-Window" . . . . . . . . . . . . . . . 304

8.1.4 Aufbau anderer Diagramme . . . . . . . . . . 309

8.1.4.1 Aufbau von Liniendiagrammen . . . . . . . . . . . 309

8.1.4.2 Aufbau von Flächendiagrammen . . . . . . . . . 315

8.1.4.3 Aufbau von Kreisdiagrammen . . . . . . . . . . . . 320

8.1.4.4 Aufbau von Boxplots, Fehlerbalken-Diagrammen und Histogrammen . . . . . . . . . . . . . 322

8.2 Ausgabe und Bereitstellung von Grafiken . . . . . . . . 330

8.2.1 Druckausgabe . . . . . . . . . . . . . . 330

8.2.1.1 Ausgabe auf den Drucker oder in eine Datei . . . . . 330

8.2.1.2 Preview für die Druckausgabe . . . . . . . . . . . 332

8.2.1.3 Änderung der Voreinstellung für die Druckausgabe . . 332

8.2.2 Ausgabe in eine Datei . . . . . . . . . . . . . 334

8.2.3 Bereitstellung von Grafiken . . . . . . . . . . 335

8.2.4 Exportierung von Grafiken .......... 336

8.2.5 Übertragung in die Zwischenablage . . . . . . . . 338

9 Das Arbeiten mit SPSS-Daten-Tabellen $\begin{array}{ll}\text { und Datenaustausch } & 339\end{array}$

9.1 Sicherung von SPSS-Daten-Tabellen . . . . . . . . 339

9.2 Wiederherstellung von SPSS-Daten-Tabellen . . . . . . 343

9.3 Zusammenfassung von SPSS-Daten-Tabellen . . . . . . . 344

9.3.1 Zusammenführung paralleler SPSS-Daten-Tabellen . . 344

9.3.2 Zusammenführung von nicht-parallelen SPSS-Daten-Tabellen . . . . . . . . . . . . . 348

9.3.3 Aneinanderreihung von gleichstrukturierten SPSS-Daten-Tabellen . . . . . . . . . . . 351

9.4 Transponieren von SPSS-Daten-Tabellen . . . . . . . . . 353

9.5 Datenaustausch mit Fremdsystemen . . . . . . . . . . 356

9.5.1 Umwandlung einer SPSS-Daten-Tabelle (Ausgabe) . . 356

9.5.2 Herstellung einer SPSS-Daten-Tabelle (Eingabe) . . . 357 
10 Speicherung von Rangwerten und Statistiken 359

10.1 Speicherung von Rangwerten . . . . . . . . . . . . 359

10.1.1 Rangwerte und Bindungen . . . . . . . . . 359

10.1.2 Transformation von Rangwerten . . . . . . . . 362

10.2 Speicherung von Statistiken . . . . . . . . . . . 366

10.2.1 Beispiel . . . . . . . . . . . . . 366

10.2.2 Indikator-Variable . . . . . . . . . . . . . 370

10.2.3 Statistiken . . . . . . . . . . . . . 371

10.2.4 Festlegung der SPSS-Daten-Tabelle . . . . . . . . 373

11 Varianzanalyse $\quad 375$

11.1 Einfaktorielle Varianzanalyse . . . . . . . . . . . . 375

11.1.1 Voraussetzungen und Nullhypothese . . . . . . . 375

11.1.2 Varianzanalyse-Tafel . . . . . . . . . . . . . 376

11.1.3 Überprüfung der Test-Voraussetzungen . . . . . . . . 378

11.1.4 Vergleiche einzelner Faktorstufen . . . . . . . . . . 379

11.1.5 "A priori"-Vergleiche . . . . . . . . . . . . . . . 384

11.1.6 Trend-Tests . . . . . . . . . . . . . . . . 385

11.1.7 Optionale Anforderungen . . . . . . . . . . 387

11.2 Mehrfaktorielle Varianzanalyse . . . . . . . . . . . . 388

11.2 .1 Beispiel . . . . . . . . . . . . . 388

11.2.2 Varianzanalyse-Tafel . . . . . . . . . . . . . 389

11.2.3 Zellenbesetzungen . . . . . . . . . . . . . 391

11.2.4 Grafische Darstellung . . . . . . . . . . . . . . 392

11.2.5 Mehr als zwei Faktoren . . . . . . . . . . . . . 394

11.2.6 Multiple Klassifikationsanalyse . . . . . . . . . . 394

11.2.7 Kovarianzanalyse . . . . . . . . . . . . . . 396

11.2.8 Optionale Anforderungen . . . . . . . . . 398

12 Nichtparametrische Testverfahren 401

12.1 Vergleich mit einer theoretischen Verteilung . . . . . . . . . 401

12.1.1 Der Chi-Quadrat-Test . . . . . . . . . . . . . 402

12.1.2 Der Binomial-Test . . . . . . . . . . . . . . 403

12.1.3 Der Kolmogorov-Smirnov-Test . . . . . . . . . . 404 
12.2 Vergleich zwischen empirisch ermittelten Verteilungen . . . 405 12.2.1 Paarvergleich bei abhängigen Stichproben . . . . . . 406 12.2.2 Vergleich mehrerer Verteilungen bei abhängigen Stichproben . . . . . . . . . . . 408

12.2.3 Verteilungs-Vergleich bei zwei unabhängigen Stichproben . . . . . . . . . 410

12.2.4 Verteilungs-Vergleich

bei mehreren unabhängigen Stichproben . . . . . . . 412

12.3 Sequenzanalyse . . . . . . . . . . . . . . 415

12.4 Optionale Anforderungen $\ldots \ldots \ldots 416$

13 Regressionsanalyse

13.1 Beschreibung der linearen Beziehung und Anpassungsgüte . . 418

13.2 Überprüfung der Voraussetzungen . . . . . . . . . . . . . 424

13.2.1 Prüfung der Linearitätsannahme, der Mittelwert- und der Varianzeigenschaft . . . . . . 424

13.2.2 Prüfung der Normalverteilungsannahme . . . . . . . 427

13.2.3 Identifikation von statistischen Ausreißern . . . . . . . 428

13.3 Statistiken und Multikollinearität . . . . . . . . . . . . 429

13.3.1 Statistiken . . . . . . . . . . . . . 429

13.3.2 Multikollinearität . . . . . . . . . . . . . 431

13.4 Ergänzung der SPSS-Daten-Tabelle . . . . . . . . . . . 433

13.5 Gewichtung und Methoden der schrittweisen Regression . . . 435

14 Analyse von Zeitreihen

14.1 Aufbau und Bearbeitung von Zeitreihen . . . . . . . . . . . . . 442

14.1.1 Eingabe von Zeitreihenwerten . . . . . . . . . . 442

14.1.2 Ersetzung von missing values . . . . . . . . . 446

14.1.3 Transformation von Zeitreihen . . . . . . . . . . 448

14.2 Anzeige des Zeitpolygons . . . . . . . . . . . . 450

14.3 Untersuchung auf Normalverteilung . . . . . . . . . . . . . . . . . . . . . . . . . .

14.4 Strukturuntersuchungen von Zeitreihen . . . . . . . . . 458

14.4.1 Autokorrelationen und Korrelogramm . . . . . . . 458

14.4.2 Partielle Autokorrelation . . . . . . . . . . . 461

14.4.3 Kreuz-Korrelation . . . . . . . . . . . . 464 
14.5 Bestimmung des Trends . . . . . . . . . . . . . . 467

14.5.1 Modellansätze . . . . . . . . . . . . . . 467

14.5.2 Prognosen . . . . . . . . . . . . . . . 471

14.5.3 Auswahl des Prognose-Stützbereichs . . . . . . . 473

14.6 Vergleich von Zeitreihenwerten . . . . . . . . . . . . 475

14.6.1 Vergleiche zwischen Zeitreihen über "High-Low-Diagramme" . . . . . . . . . . . 475

14.6.2 Vergleiche innerhalb von Zeitreihen durch "HighLow-", "Pareto-" und "Kontrollkarten-Diagramme" . 479

Anhang

487

A.1 Dateneingabe aus Text-Dateien . . . . . . . . . . 487

A.2 Dateneingabe aus Datenbanken . . . . . . . . . . . 492

A.3 Menü-gestützte Editierhilfen . . . . . . . . . . . . . 501

A.4 Symbol-Leisten . . . . . . . . . . . . . . . 503

A.5 Einfügung von SPSS-Ergebnissen in Word-Dokumente . . . . 510

A.6 Abruf von Direkthilfen und "Help-Windows" . . . . . . . . . . 514

Literaturverzeichnis

517

Index

518 